\title{
A novel classifier based on three preoperative tumor markers predicting the cancer-specific survival of gastric cancer (CEA, CA19-9 and CA72-4)
}

\author{
Jing Guo ${ }^{1,2, *}$, Shangxiang Chen ${ }^{1,2, *}$, Shun $\mathrm{Li}^{1,2, *}$, Xiaowei Sun ${ }^{1,2}$, Wei Li $^{1,2}$, Zhiwei \\ Zhou ${ }^{1,2}$, Yingbo Chen ${ }^{1,2}$ and Dazhi $\mathrm{Xu}^{1,2}$ \\ ${ }^{1}$ State Key Laboratory of Oncology in South China, Collaborative Innovation Center for Cancer Medicine, Guangzhou, China \\ ${ }^{2}$ Department of Gastric Surgery, Sun Yat-sen University Cancer Center, Guangzhou, China \\ "These authors have contributed equally to this work
}

Correspondence to: Dazhi Xu, email: xudzh@sysucc.org.cn

Keywords: tumor marker; CEA; CA 19-9; CA72-4; gastric cancer

Received: July 07, $2017 \quad$ Accepted: December 01, 2017

Published: December 14, 2017

Copyright: Guo et al. This is an open-access article distributed under the terms of the Creative Commons Attribution License 3.0 (CC BY 3.0), which permits unrestricted use, distribution, and reproduction in any medium, provided the original author and source are credited.

\section{ABSTRACT}

Several studies have highlighted the prognostic value of the individual and the various combinations of the tumor markers for gastric cancer (GC). Our study was designed to assess establish a new novel model incorporating carcino-embryonic antigen (CEA), carbohydrate antigen 19-9 (CA19-9), carbohydrate antigen 72-4 (CA72-4). A total of 1,566 GC patients (Primary cohort) between Jan 2000 and July 2013 were analyzed. The Primary cohort was randomly divided into Training set $(n=783)$ and Validation set $(n=783)$. A three-tumor marker classifier was developed in the Training set and validated in the Validation set by multivariate regression and risk-score analysis. We have identified a three-tumor marker classifier (including CEA, CA19-9 and CA72-4) for the cancer specific survival (CSS) of GC $(p<0.001)$. Consistent results were obtained in the both Training set and Validation set. Multivariate analysis showed that the classifier was an independent predictor of GC (All p value $<0.001$ in the Training set, Validation set and Primary cohort). Furthermore, when the leaveone-out approach was performed, the classifier showed superior predictive value to the individual or two of them (with the highest AUC (Area Under Curve); 0.618 for the Training set, and $\mathbf{0 . 6 2 5}$ for the Validation set), which ascertained its predictive value. Our three-tumor marker classifier is closely associated with the CSS of GC and may serve as a novel model for future decisions concerning treatments.

\section{INTRODUCTION}

Gastric cancer has been the third leading cause of cancer-related death worldwide and has a 5-year survival no more than $30 \%[1,2]$. GC patients are usually diagnosed at advanced stage and have poor survival. Despite the widely used $7^{\text {th }}$ AJCC TNM stage, the prognosis of GC may be affected by other factors like tumor differentiation, behavior and genetic abnormalities [3-5]. While they could not either work timely or easily accessible to the public. Therefore, accurate, costless and easily accessible indexes are essential for survival assessment of GC.
Serum tumor markers such as CEA, CA19-9 and CA72-4 has been demonstrated to elevate in GC patients at various stages and associated with survival [6-9]. However, their low rates of sensitivity and specificity limited their application in clinical practice. As demonstrated in previous literature, when used as a combination, these markers had the ability to increase the sensitivity and accuracy for survival prediction $[10,11]$.

In the context, we investigated the prognostic value of CEA, CA19-9 and CA72-4. Individual and different combinations of the tumor markers were all evaluated and compared. Then, we established a new combined novel model of these three markers, which could obviously 
Table 1: Baseline characteristics of training set and validation

\begin{tabular}{|c|c|c|c|}
\hline Variable & Training set & Validation set & P value \\
\hline Total study & 783 & 783 & \\
\hline Age (years) & & & 0.193 \\
\hline$<63 / \geq 63$ & $522 / 261$ & $546 / 237$ & \\
\hline Gender & & & 0.123 \\
\hline Male/Female & $500 / 283$ & $529 / 254$ & \\
\hline Differentiated type & & & 0.418 \\
\hline Well/Moderate and poor & $262 / 521$ & $247 / 536$ & \\
\hline Tumor size $\left(\mathrm{cm}^{2}\right)$ & & & 0.169 \\
\hline$(<4 / \geq 4)$ & $332 / 451$ & $359 / 424$ & \\
\hline Location & & & 0.501 \\
\hline Upper/Middle/Lower & $201 / 289 / 293$ & $207 / 267 / 309$ & \\
\hline \multicolumn{4}{|l|}{ Chemotherapy } \\
\hline YES/NO & $221 / 562$ & $211 / 572$ & 0.572 \\
\hline T stage & & & 0.593 \\
\hline $\mathrm{T} 1 / \mathrm{T} 2 / \mathrm{T} 3 / \mathrm{T} 4$ & $98 / 76 / 335 / 274$ & $89 / 85 / 352 / 257$ & \\
\hline $\mathrm{N}$ stage & & & 0.992 \\
\hline $\mathrm{N} 0 / \mathrm{N} 1 / \mathrm{N} 2 / \mathrm{N} 3$ & $222 / 174 / 190 / 197$ & $219 / 174 / 195 / 195$ & \\
\hline Metastasis & & & 1.000 \\
\hline YES/NO & $200 / 583$ & $200 / 583$ & \\
\hline AJCC stage & & & 0.566 \\
\hline I/II/III/IV & $123 / 156 / 304 / 200$ & $117 / 167 / 299 / 200$ & \\
\hline CEA (ng/ml) & $42.83 \pm 412.19$ & $9.41 \pm 31.57$ & 0.963 \\
\hline CA19-9 (U/ml) & $97.99 \pm 551.40$ & $135.31 \pm 1367.92$ & 0.216 \\
\hline CA72-4 (U/ml) & $16.78 \pm 103.13$ & $13.42 \pm 82.05$ & 0.773 \\
\hline
\end{tabular}

CEA, carcino-embryonic antigen; CA19-9, carbohydrate antigen 19-9; CA72-4, carbohydrate antigen 72-4; AJCC, American Joint Committee on Cancer.

improve the prognostic power and provide a footprint for accurate survival prediction of GC.

\section{RESULTS}

\section{Baseline characteristics}

We retrospectively studied 1,566 GC patients, which were classified in a training set $(n=783)$ and a validation set $(\mathrm{n}=783)$. The mean age was $56.3 \pm 12.6$ and $55.9 \pm 12.7$ for training set and validation set respectively. There were 500 men in the training set and 529 men in the validation set. $42.6 \%$ of the patients died when this study was completed. The 5-year overall survival was
$19.9 \%$. The median follow-up time was 32.55 months in the training set and 31.7 months in the validation set. The clinicopathologic characteristics of each set are listed in Table 1.

\section{Association between the three tumor markers and the CSS}

Cox Regression analysis showed that the serum expression levels of the three tumor markers was significantly associated with the CSS in a dose-dependent manner. These three markers were all risk factors and their high expression contributed to unfavorable survivals ( $p<0.001$ for CEA, CA19-9 and CA72-4 respectively). 
Table 2: Serum expression levels of three tumor markers and survival of GC patients in all sample sets

\begin{tabular}{|c|c|c|c|c|c|}
\hline Combined tumor marker Data set & Patients & Deaths & MST (months) & $P$ Value $^{\mathrm{a}}$ & $95 \% \mathrm{CI}$ \\
\hline \multicolumn{6}{|l|}{ CEA } \\
\hline Low, $\leq 2.48$ & 863 & 306 & 25.03 & & \\
\hline High, $>2.48$ & 703 & 337 & 18.90 & $<0.001$ & $1.509(1.293,1.762)$ \\
\hline \multicolumn{6}{|l|}{ CA19-9 } \\
\hline Low, $\leq 28.81$ & 1246 & 455 & 24.47 & & \\
\hline High, $>28.81$ & 320 & 188 & 16.30 & $<0.001$ & $1.957(1.650,2.321)$ \\
\hline \multicolumn{6}{|l|}{ CA72-4 } \\
\hline Low, $\leq 2.47$ & 1025 & 396 & 24.97 & & \\
\hline High, $>2.47$ & 541 & 247 & 17.67 & $<0.001$ & $1.417(1.209,1.662)$ \\
\hline No. of high risk tumor marker(ref:0) & 525 & 169 & 27.80 & $<0.001$ & \\
\hline 1 & 610 & 236 & 22.90 & 0.003 & $1.352(1.110,1.648)$ \\
\hline 2 & 339 & 178 & 18.13 & $<0.001$ & $2.033(1.647,2.510)$ \\
\hline 3 & 92 & 60 & 11.15 & $<0.001$ & $3.263(2.427,4.386)$ \\
\hline
\end{tabular}

CEA, carcino-embryonic antigen; CA19-9, carbohydrate antigen 19-9; CA72-4, carbohydrate antigen 72-4; No, number.

${ }^{a}$ Cox proportional hazards model.

Moreover, the median survival time (MST) was statistically significant between the different expression of the three tumor markers $(25.03$ months versus 18.90 months, $\mathrm{p}<0.001$ for CEA; 24.47 months versus 16.30 months $\mathrm{p}<0.001$ for CA19-9 and 24.97 versus 17.67 months, $\mathrm{p}<0.001$ for CA72-4). When taking the three markers into consideration simultaneously, we found that patients with higher-risk tumor markers showed worse survival than the less-risk ones. (Table 2) Similar results were also identified in both the Training and Validation set.

\section{Three-tumor marker classifier and patient CSS}

Based on the Training set, we constructed a model using these three markers by a risk score method. The multiple of the expression level of the reference value was evaluated by Cox regression and each tumor marker reached a coefficient (1.944 for CEA, 1.746 for CA199 and 1.313 for CA72-4). Then a risk score was derived by a summation of the multiple times its corresponding coefficient: Risk score $=(1.944 \times$ the multiple of CEA expression level to reference value $)+(1.746 \times$ the multiple of CA19-9 expression level to reference value) $+(1.313 \times$ the multiple of CA72-4 expression level to reference value). For instance, the reference value of tumor markers was $0-5.0 \mathrm{ng} / \mathrm{ml}$ for CEA, $0-35.0 \mathrm{U} / \mathrm{ml}$ for CA19-9 and 0-5.3 U/ml for CA72-4. When there was a GC patient with markers expression levels as follows (CEA, $10 \mathrm{ng} / \mathrm{ml}$; CA19-9, 35.0U/ml; CA72-4, 5.3U/ml), the Risk score $=1.944 \times 2+1.746 \times 1.0+1.313 \times 1.0=6.903$. The patients in the Training set were then divided into high and low risk score according to the cutoff point generated by the Youden's index method (cutoff point: Risk score=3.74). CSS of patients with low-risk score (Risk score $\leq 3.74$ ) and those with high-risk score showed obvious statistical significance. The high-risk score patients had shorter MST than the low-risk score group (MST: 15.17 months versus 28.45 months, $\mathrm{p}<0.001$ ) and increased HR (HR: $2.792,95 \%$ CI: $2.234,3.491)$ for CSS. To test the stability of the three-marker classifier, an internal validation was performed in the Validation set and the Primary set. The same cutoff points and model were applied. Similar results were obtained for which the high-risk score group still had a shorter MST (MST: 24.72 months versus 16.13 months, $\mathrm{p}<0.001$ for the Validation set; MST: 26.23 months versus 16.27 months, $\mathrm{p}<0.001$ for the Primary set) and elevated HR (HR:1.765, 95\%CI:1.418, 2.197 for the Validation set and HR: 2.244, 95\%CI:1.921, 2.621 for the Primary set, respectively).(Figure 1 and Table 3)

\section{Tumor markers classifier predicts CSS better and independently}

To investigate whether the classifier was an independent prognostic factor for $\mathrm{GC}$, univariate and multivariate analysis were performed. As illustrated in Table 4, the Risk score and the $7^{\text {th }}$ AJCC stage were all associated with the CSS in the Training set via the univariate and multivariate analysis $(p<0.001$ for both Risk score and 7th AJCC stage). Similar consistent results were observed in the Validation set, for which risk score 
was still an independent prognostic factor $(\mathrm{p}<0.001$; HR: 1.446; 95\% CI: 1.160, 1.852). Moreover, the ROC curves and Leave-one-out approach were performed to further assess the discrimination ability (Figure 2). AUCs were reached to evaluate the predictive value of our classifier. Surprisingly, the AUCs of the three-marker classifier were 0.618 ( $\mathrm{p}<0.001 ; 95 \%$ CI: $0.577,0.658$ for Training set) and 0.625 ( $\mathrm{p}<0.001 ; 95 \%$ CI: $0.584,0.666$ for Validation),
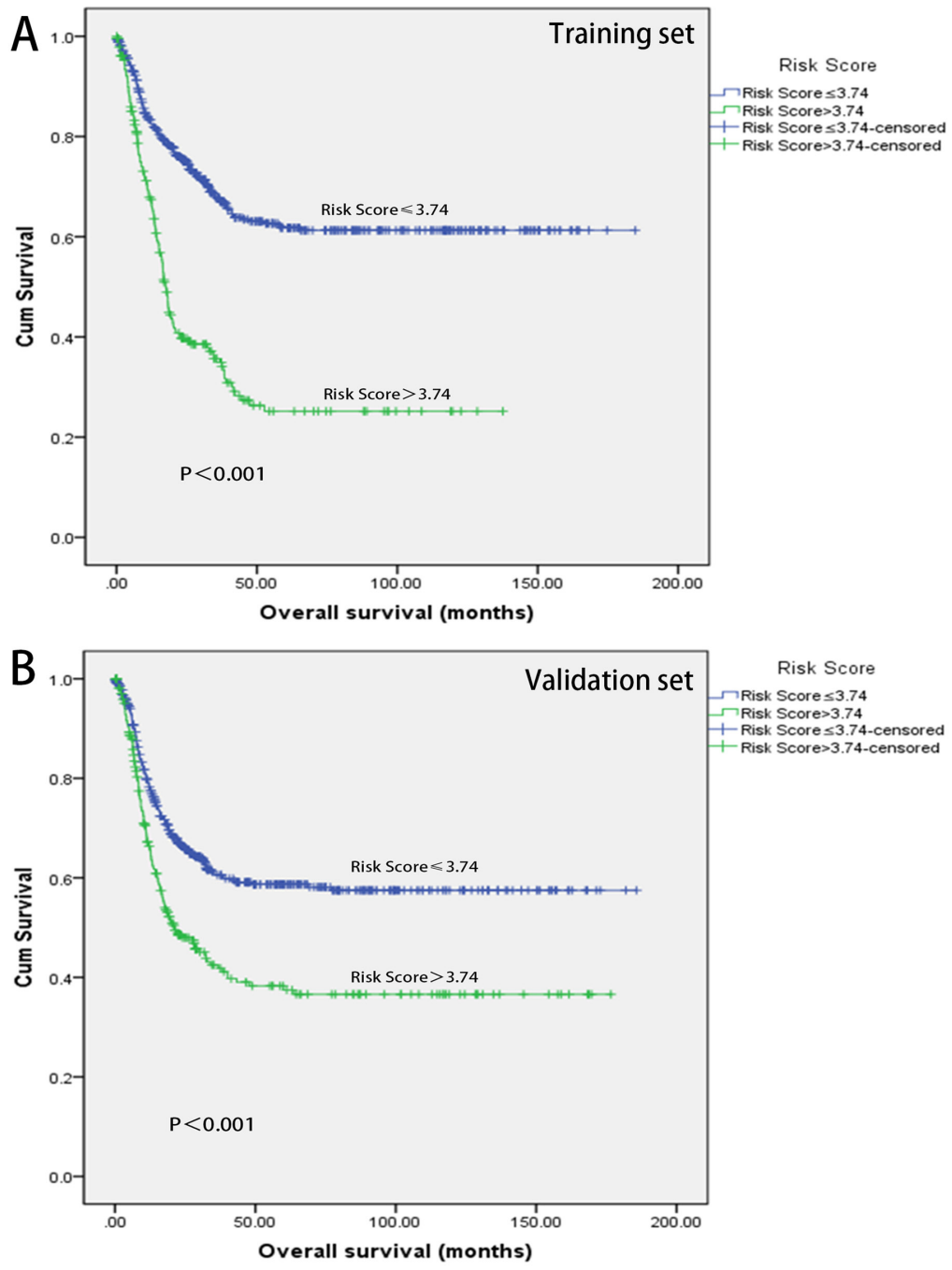

Risk Score $>3.74$-censored
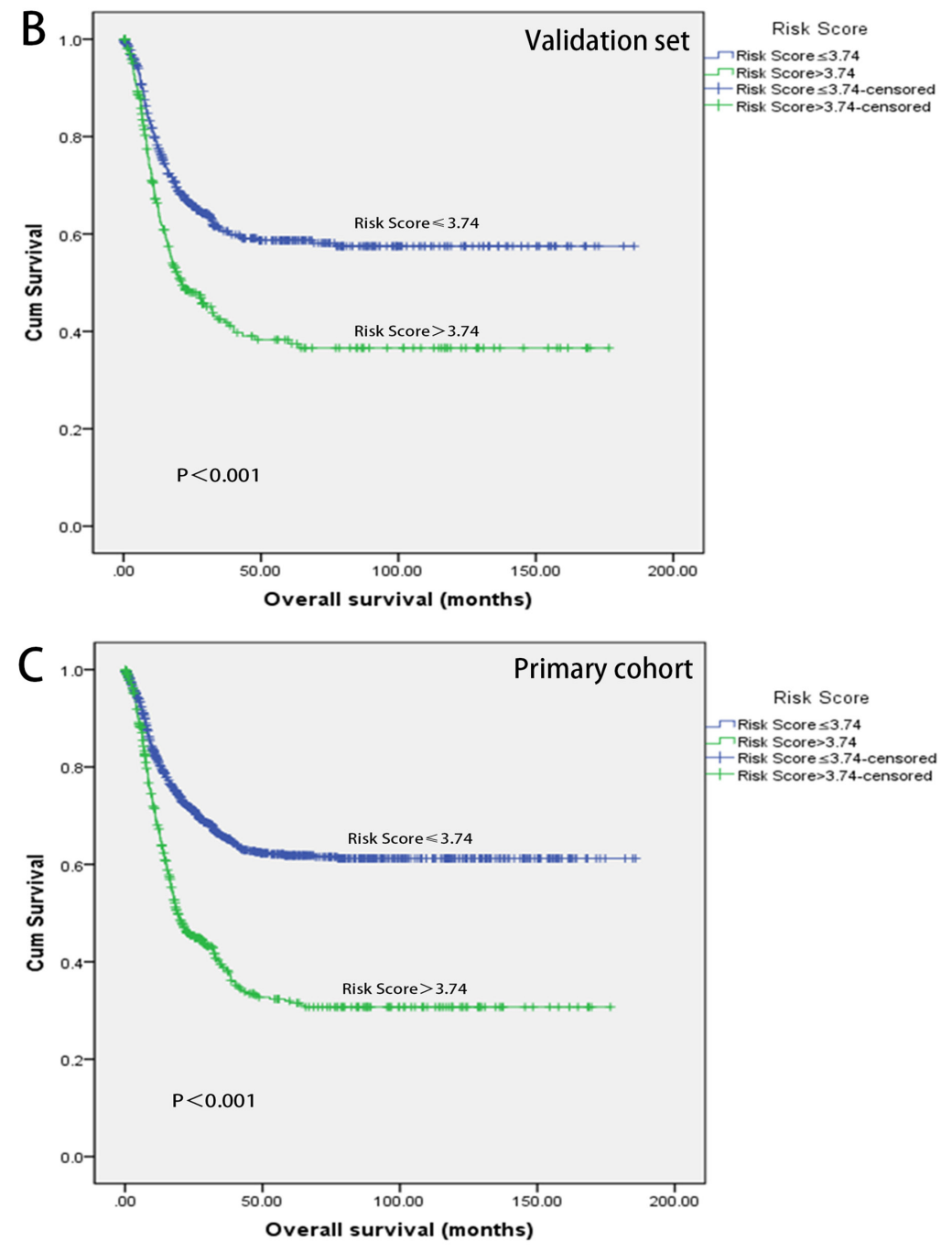

Figure 1: Kaplan-Meier analyses of overall survival. Survival analyses of training set stratified by Risk score groups (A). Survival analyses of Validation set stratified by Risk score groups (B). Survival analyses of Primary cohort stratified by Risk score groups (C). 
Table 3: Risk score and survival of GC patients in training set, validation set and primary cohort

\begin{tabular}{|c|c|c|c|c|c|}
\hline Combined tumor marker Data set & Patients & Deaths & MST (months) & $P$ Value $^{\mathrm{a}}$ & $95 \% \mathrm{CI}$ \\
\hline \multicolumn{6}{|l|}{ Training set } \\
\hline No. of patients & 783 & 316 & & & \\
\hline Low, $\leq 3.74$ & 546 & 170 & 28.45 & & \\
\hline High, $>3.74$ & 237 & 146 & 15.17 & $<0.001$ & $2.792(2.234,3.491)$ \\
\hline \multicolumn{6}{|l|}{ Validation set } \\
\hline No. of patients & 783 & 327 & & & \\
\hline Low, $\leq 3.74$ & 512 & 183 & 24.72 & & \\
\hline High, $>3.74$ & 271 & 144 & 16.13 & $<0.001$ & $1.765(1.418,2.197)$ \\
\hline \multicolumn{6}{|l|}{ Primary cohort } \\
\hline No. of patients & 1566 & 643 & & & \\
\hline Low, $\leq 3.74$ & 1021 & 328 & 26.23 & & \\
\hline High, $>3.74$ & 545 & 315 & 16.27 & $<0.001$ & $2.244(1.921,2.621)$ \\
\hline
\end{tabular}

higher than any other individuals or combinations. (Table 5)

\section{DISCUSSION}

In the present study, we developed and validated a new three-tumor marker classifier (CEA, CA19-9 and CA72-4) for GC by the risk-score method [12]. A significant survival difference was detected between low and high-risk score GC patients. The presence of a high risk tended to associate with unfavorable CSS. When compared to the individual and various combinations of these markers, our classifier showed the best discrimination ability in survival assessment. Herein, we proposed a novel three-tumor marker classifier which could assess survival more effectively.

It is widely known that the serum expression levels of tumor markers (CEA, CA19-9 and CA72-4) is usually increased upon $\mathrm{GC}$ diagnosis and has been found to affect prognosis $[8,13,14]$. As reported, CEA played a critical role in programmed cell death and cell adhesion, high pretherapeutic level of CEA was correlated with the stage of the disease, especially in patients with peritoneal serous carcinoma $[15,16]$. CA19-9 correlated with lymph node involvement and was an independent predictive factor in metastatic or recurrent GC patients $[17,18]$. CA72-4 was demonstrated to have the highest overall positive rate [6]. As a result, the Japanese Public Health Insurance System even covers the costs of monitoring patients with GC by serum tumor markers.

Despite the important predictive value of these tumor markers, limited sensitivity and specificity restricted their applications in daily practice. Previous studies have identified the superiority of combined tumor markers for the individuals $[11,19,20]$. However, few of them had reached a satisfied result due to the intrinsic deficiency in the markers. To improve the discrimination ability, various tumor markers were taken into consideration simultaneously in this study. We firstly investigated the relationship between the serum expression levels of tumor marker (CEA, CA19-9 and CA72-4) and the CSS of the patients treated at our hospital. High expression level groups showed better survival than the low expression groups, which was consistent with recent published studies [21-24]. Furthermore, patients with two or more high risk tumor markers had increased probability of shorter survival than those with one or less, providing the potential role of the combined tumor markers in survival assessment.

To established a model which would take tumor markers (CEA, CA19-9 and CA72-4) into account comprehensively, we proposed a risk score. A linear combination weighted by the regression coefficient was firstly used to evaluate the individual role of tumor markers in our study. Our classifier was further observed to be an independent prognostic predictor for tumor classification and demonstrated to have a higher AUC than other biomarkers or combinations, which thereby indicated a better discrimination ability.

Similar studies were conducted recently and they were mainly restricted by their limited sample to draw reliable results. For example, $\mathrm{He}$ et al found the combination of AFP, CEA, CA125 and CA19-9 increased the sensitivity for the diagnosis of GC using a cohort of 149 patients [20]. Sun et al performed a retrospective review in 184 GC patients and found CEA, CA19-9, CA72-4, 
Table 4: Univariate and multivariate analysis of training set and validation

\begin{tabular}{|c|c|c|c|c|}
\hline \multirow{2}{*}{ Variable } & \multicolumn{2}{|c|}{ Univariate analysis } & \multicolumn{2}{|c|}{ Multivariate analysis } \\
\hline & $P^{a}$ value & Hazard ratio( $95 \% \mathrm{CI})$ & P value $^{a}$ & Hazard ratio $(95 \% \mathrm{CI})$ \\
\hline \multicolumn{5}{|l|}{ Training set } \\
\hline $7^{\text {th }}$ AJCC stage (ref: I) & $<0.001$ & $2.598(2.256,2.991)$ & $<0.001$ & \\
\hline II & & & 0.003 & $3.810(1.573,9.232)$ \\
\hline III & & & $<0.001$ & $12.926(5.699,29.318)$ \\
\hline IV & & & $<0.001$ & $24.239(10.624,55.301)$ \\
\hline Differentiated type & 0.009 & $1.376(1.084,1.748)$ & 0.042 & $1.284(1.009,1.635)$ \\
\hline Risk score & $<0.001$ & $2.082(1.673,2.592)$ & 0.015 & $1.323(1.056,1.658)$ \\
\hline \multicolumn{5}{|l|}{ Validation set } \\
\hline $7^{\text {th }}$ AJCC stage (ref: I) & $<0.001$ & $2.598(2.256,2.991)$ & $<0.001$ & \\
\hline II & & & 0.003 & $3.466(1.527,7.869)$ \\
\hline III & & & $<0.001$ & $9.610(4.472,20.649)$ \\
\hline IV & & & $<0.001$ & $19.974(9.271,43.0 .32)$ \\
\hline Location (ref: Lower) & $<0.001$ & $2.535(2.203,2.917)$ & 0.004 & \\
\hline Upper & & & 0.027 & $1.369(1.036,1.809)$ \\
\hline Middle & & & 0.470 & $0.895(0.662,1.209)$ \\
\hline Age & 0.001 & $1.486(1.181,1.869)$ & 0.001 & $1.498(1.185,1.892)$ \\
\hline Risk score & $<0.001$ & $2.417(1.933,3.023)$ & 0.001 & $1.446(1.160,1.852)$ \\
\hline
\end{tabular}

AJCC, American Joint Committee on Cancer.

${ }^{a}$ Cox proportional hazards model.
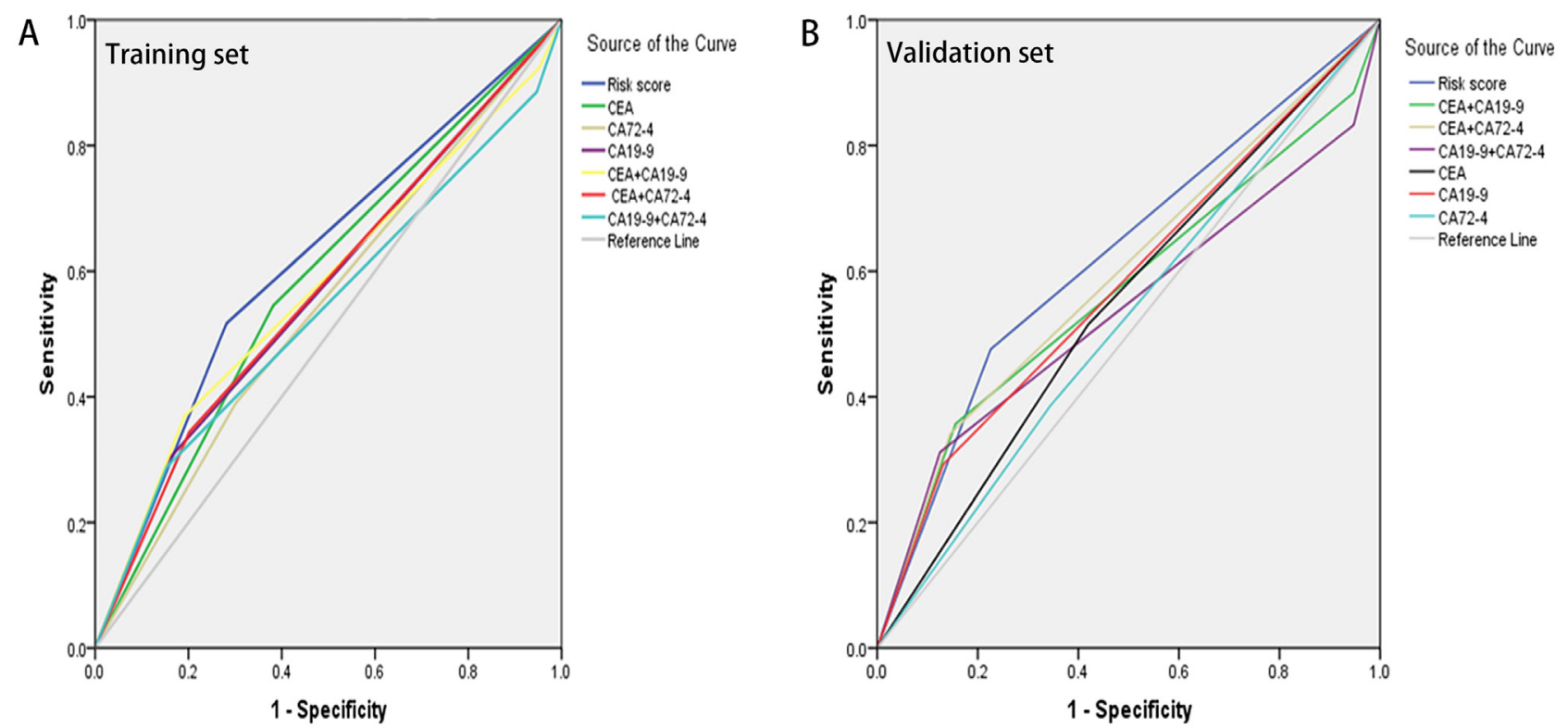

Figure 2: The predictive ability of the Risk score compared by ROC curves. Comparison in the Training set (A). Comparison in the Validation set (B). 
Table 5: Comparison of the AUCs for the different classifiers

\begin{tabular}{lccc}
\hline & AUC & $\mathbf{9 5 \%}$ CI & $\boldsymbol{P}$ Value \\
\hline Training set & & & $<0.001$ \\
Risk score & 0.618 & $(0.577-0.658)$ & $<0.001$ \\
CEA & 0.582 & $(0.541-0.623)$ & 0.001 \\
CA19-9 & 0.571 & $(0.530-0.613)$ & 0.036 \\
CA72-4 & 0.544 & $(0.503-0.586)$ & 0.001 \\
CEA+CA19-9 & 0.573 & $(0.531-0.615)$ & 0.001 \\
CEA+CA72-4 & 0.571 & $(0.530-0.612)$ & 0.072 \\
CA19-9+CA72-4 & 0.538 & $(0.495-0.580)$ & $<0.001$ \\
Validation set & & & 0.024 \\
Risk score & 0.625 & $(0.584-0.666)$ & $<0.001$ \\
CEA & 0.548 & $(0.506-0.589)$ & 0.328 \\
CA19-9 & 0.581 & $(0.539-0.622)$ & 0.001 \\
CA72-4 & 0.521 & $(0.479-0.562)$ & $<0.001$ \\
CEA+CA19-9 & 0.569 & $(0.525-0.612)$ & 0.071 \\
CEA+CA72-4 & 0.598 & $(0.556-0.640)$ & \\
CA19-9+CA72-4 & 0.538 & $(0.494-0.583)$ & \\
\hline
\end{tabular}

CEA, carcino-embryonic antigen; CA19-9, Carbohydrate Antigen 19-9; CA72-4, Carbohydrate Antigen 72-4.

and CA125 could be used to evaluate the diagnosis and prognosis value for GC patients [19]. Clinically it would be partly biased to reach a firm conclusion based on such a mono-institutional small sized population. In contrast, our study was based on a much larger cohort and demonstrated reliable results. In addition, a validation method was also used in this study unlike the previously published ones. We grouped the population randomly into two samples to avoid the heterogeneity of data and additionally to justify the universal usefulness of our model. Therefore, our larger sample size provided stronger evidence for the clinical value of the risk score.

Given its high efficiency, low cost and convenience, it is easy to be performed routinely in practice. GC is a malignant tumor which metastases early by peritoneum, lymphatic system, and blood. Recurrences are very common after surgery. Nearly half of GC patients with radical gastrectomy relapse within 2 years [25]. According to our results, high-risk score indicates a reduction in overall survival. The risk score could help to make more considerate decisions in stage evaluation, adjuvant therapy selection and follow-up arrangements.

Apart from the satisfactory results in our study, it was also bound to some limitations. First, as a retrospective single-institution database, whether the results could be applicable to other populations requires further validation, although an internal validation was performed. Second, it may at times be mathematically time-consuming to assess
GC survival with such a formula and in the short future we still actively working to devise an online electronic downloadable conversion method which would be more practical. Finally, if possible, when post-operative serum tumor marker data were incorporated, our study would have been more valuable.

In summary, these results clearly indicated that, although one could distinguish patients with different prognosis, combined classifier worked better. It is worth noting that our combined classifier may play a critical role in GC prognosis estimation, personalized therapy and therapeutic evaluation. Patients with elevated risk score are suggested to receive closer follow-up. Further validations are warranted for wider application.

\section{MATERIALS AND METHODS}

\section{Study population and data collection}

The data of 1,566 patients hospitalized at the Department of Gastric Surgery, Sun Yat-Sen Cancer Center, Guangzhou, China from January 2000 to July 2013 were analyzed. All surgically treated patients with pathologically or histologically proven GC were included. all the patients had received surgery directly without receiving any sort of neoadjuvant chemotherapy. Patients were excluded if demonstrated other malignancies, organ insufficiencies or experienced an acute event (acute or 
chronic inflammation, immune disease, hematological disease, or liver disease that could influence the level of tumor markers). The data analyzed were age, gender, differentiated type, tumor location, tumor size, chemotherapy status, depth of wall invasion, lymph node metastasis, remote metastasis and the expression levels of these three tumor markers. Patients were staged according to the 7th edition AJCC (American Joint Committee on Cancer) Tumor-Node-Metastasis(TNM) criteria [26]. Venous blood sample for marker determination was separated by centrifugation, and aliquots were stored at $-20{ }^{\circ} \mathrm{C}$ until assayed, with informed consent.

\section{Statistical analysis}

1,566 cases were randomly classified as a Training $(n=783)$ set and a Validation set $(n=783)$ by using computer-generated random numbers for survival analysis. In the training set, we used univariate Cox regression analysis to evaluate the association between the expression level of tumor maker and the survival [27]. Tumor markers with HR for death less than 1 was defined as the protective factors, while those with HR values greater than 1 were classified as the risk factors. For tumor markers that significantly correlated with survival, we assigned each patient a risk score calculated by a linear combination of the multiple generated from the division between the expression level of the marker and the reference value, weighted by the regression coefficient [28].

To further test the predictive value of the risk score, the same algorithm was validated in Validation set and the Primary cohort. Leave-one-out approach was used to compare the predictive value of our model with various combinations and the individuals. Data analysis was established by the Receiver Operating Characteristic (ROC) curves, the Kaplan-Meier method, log-rank test, Kruskal-Wallis test, Chi-square test and Cox proportional hazard regression models.

The primary endpoint of this study was cancerrelated death. The follow-up duration was defined as the interval from the time of surgery to the last date of followup, and the cancer specific survival (CSS) was defined as the time between the operation and cancer-related death or the last follow-up. Of note, the last follow-up was up to Jan 2016.

All statistical analysis was performed by the statistical software package for social sciences version 21.0 (SPSS, Chicago, IL). $\mathrm{P}<0.05$ was considered as statistically significant.

\section{CONCLUSIONS}

To our knowledge, it is the first time that the threetumor marker classifier is proposed and validated in such a large cohort. As our results described, the combined classifier was found to be an independent prognostic factor for GC patients, and its predictive ability was superior to the individual and other various combinations of tumor markers. Regarding its efficiency, cheapness and availability, it will be of great importance in survival evaluation and therapeutic strategy decision.

\section{ACKNOWLEDGMENTS}

All work was completed at the department of gastric pancreatic surgery, Cancer Center, Sun Yat-Sen University, Guangzhou, China. We promise that this paper has not been published or contributed to other Journals so far, all authors listed had made contribution in preparing this paper and reached an agreement with the content of this paper. No other persons participated in had been left out.

\section{CONFLICTS OF INTEREST}

The authors have no potential conflicts of interest.

\section{REFERENCES}

1. DeSantis CE, Lin CC, Mariotto AB, Siegel RL, Stein KD, Kramer JL, Alteri R, Robbins AS, Jemal A. Cancer treatment and survivorship statistics, 2014. CA Cancer J Clin. 2014; 64: 252-71. https://doi.org/10.3322/caac.21235.

2. Ferlay J, Soerjomataram I, Dikshit R, Eser S, Mathers C, Rebelo M, Parkin DM, Forman D, Bray F. Cancer incidence and mortality worldwide: sources, methods and major patterns in GLOBOCAN 2012. Int J Cancer. 2015; 136: E359-86. https://doi.org/10.1002/ijc.29210.

3. Vallbohmer D, Drebber U, Schneider PM, Baldus S, Bollschweiler E, Brabender J, Warnecke-Eberz U, Monig $\mathrm{S}$, Holscher AH, Metzger R. Survivin expression in gastric cancer: association with histomorphological response to neoadjuvant therapy and prognosis. J Surg Oncol. 2009; 99: 409-13. https://doi.org/10.1002/jso.21271.

4. Li X, Zhang Y, Zhang Y, Ding J, Wu K, Fan D. Survival prediction of gastric cancer by a seven-microRNA signature. Gut. 2010; 59: 579-85. https://doi.org/10.1136/ gut.2008.175497.

5. Ahn HS, Lee HJ, Yoo MW, Jeong SH, Park DJ, Kim HH, Kim WH, Lee KU, Yang HK. Changes in clinicopathological features and survival after gastrectomy for gastric cancer over a 20-year period. Br J Surg. 2011; 98: 255-60. https://doi.org/10.1002/bjs.7310.

6. Kim DH, Oh SJ, Oh CA, Choi MG, Noh JH, Sohn TS, Bae JM, Kim S. The relationships between perioperative CEA, CA 19-9, and CA 72-4 and recurrence in gastric cancer patients after curative radical gastrectomy. J Surg Oncol. 2011; 104: 585-91. https://doi.org/10.1002/jso.21919.

7. Cainap C, Nagy V, Gherman A, Cetean S, Laszlo I, Constantin AM, Cainap S. Classic tumor markers in gastric cancer. Current standards and limitations. Clujul Med. 2015; 88: 111-5. https://doi.org/10.15386/cjmed-409. 
8. Deng K, Yang L, Hu B, Wu H, Zhu H, Tang C. The prognostic significance of pretreatment serum CEA levels in gastric cancer: a meta-analysis including 14651 patients. PLoS One. 2015; 10: e0124151. https://doi.org/10.1371/ journal.pone.0124151.

9. Zhou YC, Zhao HJ, Shen LZ. Preoperative serum CEA and CA19-9 in gastric cancer--a single tertiary hospital study of 1,075 cases. Asian Pac J Cancer Prev. 2015; 16: 2685-91.

10. Yin LK, Sun XQ, Mou DZ. Value of combined detection of serum CEA, CA72-4, CA19-9 and TSGF in the diagnosis of gastric cancer. Asian Pac J Cancer Prev. 2015; 16: 3867-70.

11. Yang AP, Liu J, Lei HY, Zhang QW, Zhao L, Yang GH. CA72-4 combined with CEA, CA125 and CA19-9 improves the sensitivity for the early diagnosis of gastric cancer. Clin Chim Acta. 2014; 437: 183-6. https://doi.org/10.1016/j. cca.2014.07.034.

12. Chen HY, Yu SL, Chen CH, Chang GC, Chen CY, Yuan A, Cheng CL, Wang CH, Terng HJ, Kao SF, Chan WK, Li HN, Liu CC, et al. A five-gene signature and clinical outcome in non-small-cell lung cancer. N Engl J Med. 2007; 356: 11-20. https://doi.org/10.1056/NEJMoa060096.

13. Chen XZ, Zhang WK, Yang K, Wang LL, Liu J, Wang L, Hu JK, Zhang B, Chen ZX, Chen JP, Zhou ZG, Mo $\mathrm{XM}$. Correlation between serum $\mathrm{CA} 724$ and gastric cancer: multiple analyses based on Chinese population. Mol Biol Rep. 2012; 39: 9031-9. https://doi.org/10.1007/ s11033-012-1774-X.

14. Ikeda Y, Mori M, Adachi Y, Matsushima T, Sugimachi $\mathrm{K}$, Saku M. Carcinoembryonic antigen (CEA) in stage IV gastric cancer as a risk factor for liver metastasis: a univariate and multivariate analysis. J Surg Oncol. 1993; 53: 235-8.

15. Tellez-Avila FI, Garcia-Osogobio SM. [The carcinoembryonic antigen: apropos of an old friend]. [Article in Spanish]. Rev Invest Clin. 2005; 57: 814-9.

16. Zhang YH, Li Y, Chen C, Peng CW. Carcinoembryonic antigen level is related to tumor invasion into the serosa of the stomach: study on 166 cases and suggestion for new therapy. Hepatogastroenterology. 2009; 56: 1750-4.

17. Dilege E, Mihmanli M, Demir U, Ozer K, Bostanci O, Kaya C, Aksakal O, Sakiz D. Prognostic value of preoperative CEA and CA 19-9 levels in resectable gastric cancer. Hepatogastroenterology. 2010; 57: 674-7.

18. Jo JC, Ryu MH, Koo DH, Ryoo BY, Kim HJ, Kim TW, Choi KD, Lee GH, Jung HY, Yook JH, Oh ST, Kim BS, Kim JH, et al. Serum CA 19-9 as a prognostic factor in patients with metastatic gastric cancer. Asia Pac J Clin Oncol. 2013; 9: 324-30. https://doi.org/10.1111/ajco.12019.
19. Sun Z, Zhang N. Clinical evaluation of CEA, CA199, CA72-4 and CA125 in gastric cancer patients with neoadjuvant chemotherapy. World J Surg Oncol. 2014; 12: 397. https://doi.org/10.1186/1477-7819-12-397.

20. He CZ, Zhang KH, Li Q, Liu XH, Hong Y, Lv NH. Combined use of AFP, CEA, CA125 and CA19-9 improves the sensitivity for the diagnosis of gastric cancer. BMC Gastroenterol. 2013; 13: 87. https://doi. org/10.1186/1471-230x-13-87.

21. Shimada H, Noie T, Ohashi M, Oba K, Takahashi Y. Clinical significance of serum tumor markers for gastric cancer: a systematic review of literature by the Task Force of the Japanese Gastric Cancer Association. Gastric Cancer. 2014; 17: 26-33. https://doi.org/10.1007/s10120-013-0259-5.

22. Ikeguchi M, Katano K, Saitou H, Tsujitani S, Maeta M, Kaibara N. Pre-operative serum levels of CA72-4 in patients with gastric adenocarcinoma. Hepatogastroenterology. 1997; 44: 866-71.

23. Marrelli D, Roviello F, De Stefano A, Farnetani M, Garosi L, Messano A, Pinto E. Prognostic significance of CEA, CA 19-9 and CA 72-4 preoperative serum levels in gastric carcinoma. Oncology. 1999; 57: 55-62. https://doi. org/12001.

24. Carpelan-Holmstrom M, Louhimo J, Stenman UH, Alfthan H, Haglund C. CEA, CA 19-9 and CA 72-4 improve the diagnostic accuracy in gastrointestinal cancers. Anticancer Res. 2002; 22: 2311-6.

25. Noh S, Park S, Yang H, Chung H, Chung I, Kim S, Kim H, Choi J, Kim H, Yu W, Lee J, Shin D, Ji J, et al. Adjuvant capecitabine plus oxaliplatin for gastric cancer after D2 gastrectomy (CLASSIC): 5-year follow-up of an openlabel, randomised phase 3 trial. Lancet Oncol. 2014; 15: 1389-96.

26. Washington K. 7th edition of the AJCC cancer staging manual: stomach. Ann Surg Oncol. 2010; 17: 3077-9. https://doi.org/10.1245/s10434-010-1362-z.

27. Lachin JM. Sample size evaluation for a multiply matched case-control study using the score test from a conditional logistic (discrete Cox $\mathrm{PH}$ ) regression model. Stat Med. 2008; 27: 2509-23. https://doi.org/10.1002/sim.3057.

28. Lossos IS, Czerwinski DK, Alizadeh AA, Wechser MA, Tibshirani R, Botstein D, Levy R. Prediction of survival in diffuse large-B-cell lymphoma based on the expression of six genes. N Engl J Med. 2004; 350: 1828-37. https://doi. org/10.1056/NEJMoa032520. 